\section{Mécanique et division cellulaire}

\section{Contrôle temporel de l'abscission}

Julie Lafaurie-Janvore ${ }^{1}$, Matthieu Piel ${ }^{2}$
${ }^{\text {l} L a b o r a t o i r e ~ d ' h y d r o d y n a m i q u e ~(L a d H y X), ~}$ UMR 7646 CNRS École Polytechnique, route de Saclay, 91128 Palaiseau Cedex, France.

${ }^{2}$ Institut Curie, CNRS UMR 144, 26, rue d'Ulm, 75248 Paris Cedex 05, France.

julie.lafaurie@ladhyx.polytechnique.fr
> Au cours de la division cellulaire, une cellule mère se divise en deux cellules filles toutes deux identiques à la cellule mère. La cytocinèse est la dernière étape de la division permettant, après la séparation des chromatides, la séparation du cytoplasme de la cellule mère. Il est essentiel que la cytocinèse soit finement régulée, à la fois dans le temps et dans l'espace, afin d'assurer une division sans défaut et une transmission fidèle du patrimoine génétique de la cellule mère aux deux cellules filles. En effet, il a été montré depuis les années 1970 qu'un défaut de cytocinèse peut être hautement tumorigène [1], et de nombreuses maladies sont associées à des défauts de cytocinèse [2]. On peut citer par exemple, outre le cancer, diverses maladies du sang, le syndrome de Lowe ${ }^{l}$, ou encore la dégénérescence maculaire liée à l'âge (DMLA). Ainsi, comprendre comment la cellule se divise et comment ce processus est contrôlé représente un enjeu considérable pour la recherche biomédicale.

\section{La formation du pont intercellulaire} Dans les cellules animales, la cytocinèse commence dès l'anaphase et s'effectue par pincement équatorial du cytoplasme présent entre les deux jeux d'ADN fils. La contraction d'un anneau moléculaire aboutit à la formation d'un fin pont cytoplasmique reliant les deux cellules filles, appelé «pont intercellulaire».

\footnotetext{
${ }^{1}$ Le syndrome de Lowe, ou syndrome oculo-cérébro-rénal, est une maladie multisystémique caractérisée par des anomalies des yeux, du système nerveux et des reins. C'est une maladie rare de transmission récessive liée au chromosome $X$, due à une mutation du gène OCRLl, situé en Xq26.1 et codant une phosphatidylinositol 4,5-bisphosphate-5-phosphatase (Ptdlns [4,5]P2) (source Orphanet).
}

Il ne mesure qu'à peine une dizaine de microns de longueur et un à deux microns de diamètre, ce qui est suffisant pour laisser passer des petites molécules d'une cellule fille à une autre. Ce pont est constitué majoritairement de microtubules issus du fuseau mitotique et compactés lors de la contraction de l'anneau. La membrane plasmique, quant à elle, est continue autour des cellules filles et fortement attachée aux microtubules du pont intercellulaire. Le pont comporte également une pièce centrale organisatrice, le midbody.

Mécanistique de la coupure du pont À la fin de la cytocinèse, l'abscission consiste en la coupure du pont intercellulaire reliant les cellules à la fin de la division, définissant ainsi deux cellules filles définitivement séparées (Figure 1). Cette ultime étape de la division cellulaire a longtemps été considérée comme un événement simple et banal, résultant de la rupture aléatoire du pont intercellulaire. Pourtant, les travaux de recherche de ces dix dernières années ont prouvé que l'abscission consiste en une coupure précise du pont - et non une rupture - orchestrée par une machinerie moléculaire complexe et soumise à une régulation très stricte.

Au cœur de cette machinerie se trouvent les complexes protéiques ESCRT ou endosomal sorting complex required for transport. Ils ont d'abord été découverts pour leur rôle dans la régulation du trafic vésiculaire des endosomes, d'où leur nom, mais sont impliqués dans de nombreux processus cellulaires, et en particulier dans la fission membranaire. Ils sont classés en cinq grands groupes:
ESCRT-0, I, II, III, et protéines de désassemblage. Le sous-groupe des ESCRT-III est en particulier impliqué dans la fission membranaire lors du bourgeonnement des virus (comme le VIH, virus de l'immunodéficience humaine), lors de la formation des corps multivésiculaires, et lors de l'abscission en fin de cytocinèse. Après la contraction de l'anneau en début de cytocinèse, les ESCRT-I sont recrutés au niveau de la pièce centrale du pont, le midbody, et recrutent à leur tour les ESCRT-III (Figure 2). Ces derniers induisent la coupure des microtubules par la protéine Spastine [3] et, de façon simultanée, s'autoassemblent en filaments hélicoïdaux pour permettre la fission de la membrane plasmique $[4,5]$.

\section{Le contrôle temporel de la fin de la division cellulaire}

Lorsque I'on observe le déroulement dans le temps des différentes étapes de la cytocinèse, il est étonnant de remarquer que la contraction de l'anneau en début de cytocinèse est assez rapide (quelques minutes) et d'une durée constante quelle que soit la lignée étudiée, alors que le temps nécessaire aux cellules filles pour couper le pont intercellulaire est beaucoup plus long (jusqu'à plusieurs heures) et surtout très variable d'une lignée cellulaire à une autre, parfois même d'une cellule à une autre au sein d'une même lignée cellulaire. Comme toutes les étapes de la division, l'abscission doit être coordonnée dans le temps avec les autres étapes, notamment avec les événements plus précoces de la cytocinèse. L'abscission est également une étape 


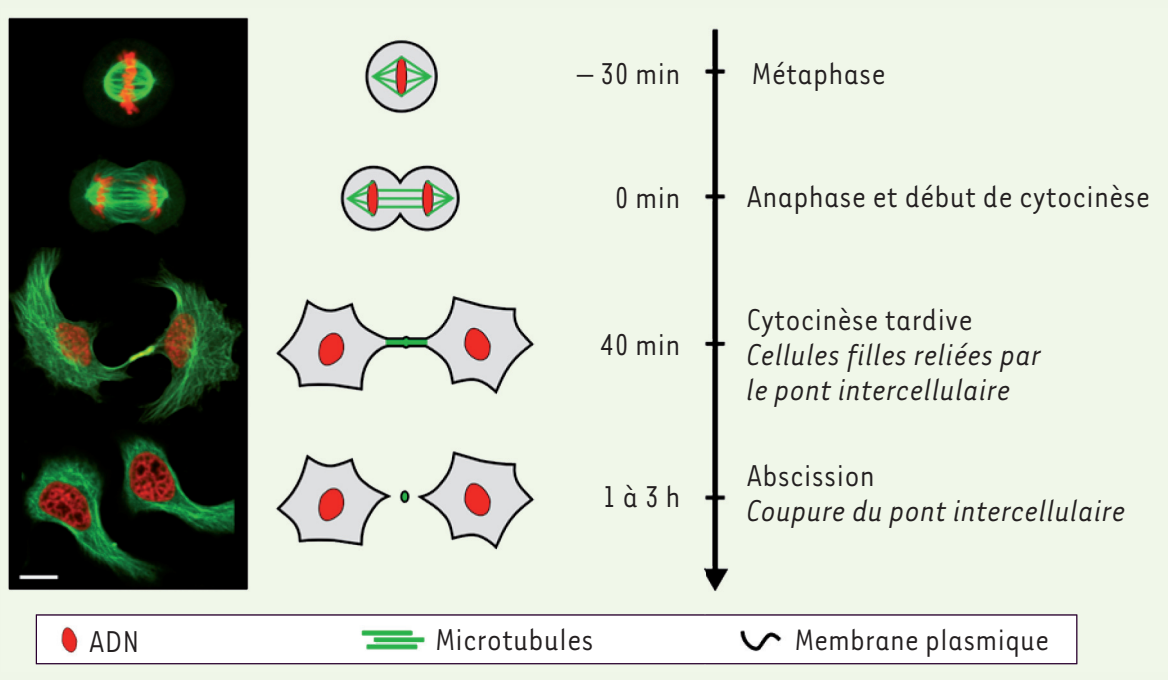

Figure 1. Division cellulaire et cytocinèse. Les différentes étapes sont illustrées à gauche par des images en microscopie optique confocale de cellules HeLa exprimant des marqueurs de microtubules (vert) et d'ADN (rouge). La barre d'échelle est de $10 \mu \mathrm{m}$. À droite, l'échelle de temps souligne la longueur et la variabilité du temps nécessaire à la coupure du pont intercellulaire reliant les deux cellules filles.

par les cellules filles sur le pont intercellulaire. En effet, lorsque les deux cellules se séparent à la fin de la division, elles migrent en s'éloignant l'une de l'autre, ce qui tend le pont qui les relie. Nous avons tout d'abord mesuré que le pont est soumis à des forces de tension de l'ordre du nanonewton. Nous avons ensuite confirmé que les cellules se séparant plus rapidement après la division tirent en effet plus fort sur le pont intercellulaire. De plus, l'analyse du mouvement des cellules filles après la division a révélé que plus les cellules se séparent vite, plus l'abscission est retardée, c'est-à-dire que, contre-intuitivement, plus les cellules filles tirent sur le pont, plus elles mettent du temps à le couper. Enfin, nous avons mon-

irréversible puisqu'aucun retour en arrière n'est possible une fois le pont coupé. Il est donc essentiel pour les cellules filles de s'assurer qu'elle intervient au bon moment, et en particulier pas trop tôt.

Le premier exemple de régulation de l'abscission que nous citerons se situe dans le contexte de la spermatogenèse. Chez la souris, il a été montré que les cellules germinales sont connectées par un large pont cytoplasmique formé à la suite d'une division dont l'abscission est inhibée. Ce syncytium peut contenir jusqu'à une centaine de cellules qui se différencient de façon parfaitement synchrone grâce aux ponts intercellulaires les connectant. La protéine TEX14 (testis-expressed gene 14), exprimée spécifiquement dans ce tissu, empêche le recrutement des ESCRT-I au niveau du pont, inhibant de façon précoce le processus d'abscission [6].

Une autre régulation temporelle de l'abscission, somatique cette fois, a été décrite lors d'une mauvaise ségrégation de I'ADN. Le checkpoint d'abscission [7-9] est un mécanisme retardant l'abscission lorsque de I'ADN est présent dans le pont intercellulaire, témoin d'une ségrégation anormale en anaphase. II permet de donner du temps aux cellules filles pour réparer cette erreur et éviter ainsi une coupure aléatoire du brin d'ADN présent dans le pont. Au niveau moléculaire, cette régulation s'effectue grâce aux protéines Aurora $B$ et CHMP4C (chromatin-modifying protein/charged multivesicular body protein [CHMP] family) qui modifient I'organisation des ESCRT-III au niveau du midbody, inhibant ainsi l'abscission.

\section{La régulation de l'abscission} par la tension entre cellules filles

De façon plus générale, il est très probable qu'une régulation temporelle constitutive garantisse que l'abscission soit déclenchée au moment adéquat, même lorsque la ségrégation de I'ADN ne présente pas de défaut. Dans le cadre d'un travail récemment publié dans Science [10], nous avons souhaité étudier les facteurs environnementaux qui pourraient retarder ou induire l'abscission des cellules. Nous nous sommes intéressés en particulier au rôle des forces de traction exercées tré qu'une relâche de la tension dans le pont induit rapidement la formation de la structure hélicoïdale d'ESCRT-III, suivie de l'abscission. Finalement, nous proposons un modèle selon lequel la machinerie moléculaire de l'abscission - essentiellement les ESCRT-III - est perturbée par une trop forte tension dans le pont intercellulaire, conduisant à un retard d'abscission lorsque les cellules filles se séparent rapidement. À l'inverse, une relâche de tension au niveau du pont, par exemple à la suite d'un mouvement des cellules filles l'une vers l'autre, induit l'abscission.

\section{Conclusion}

Nos travaux récents montrent pour la première fois que la régulation temporelle de l'abscission est assurée par l'environnement des cellules filles, en particulier par les forces exercées entre les deux cellules au niveau du pont intercellulaire : une tension dans le pont retarde l'abscission, alors qu'une diminution de la tension dans le pont induit l'abscission. II est intéressant de noter que, tout comme la régulation de l'abscission lors de la spermatogenèse ou 


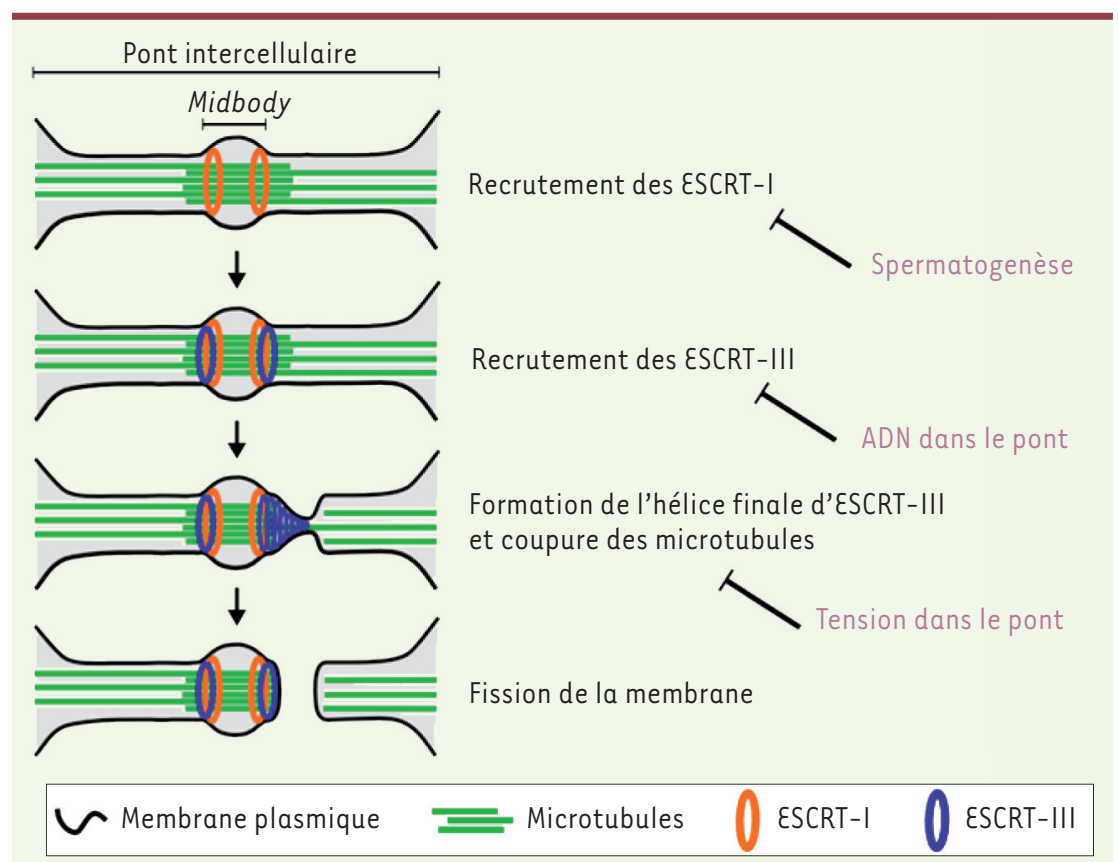

Figure 2. Le processus d'abscission et ses régulations. La pièce centrale du pont intercellulaire, le midbody, agit comme une plateforme de recrutement de la machinerie moléculaire nécessaire à l'abscission, les complexes protéiques ESCRT. Les régulations temporelles de l'abscission (à droite) retardent ou empêchent le fonctionnement des ESCRT à différents niveaux du processus d'abscission.

lors de la présence d'ADN dans le pont intercellulaire, la régulation temporelle de l'abscission par la tension entre les cellules filles s'effectue aussi via les complexes ESCRT, mais chacune de ces conditions agit à un niveau différent du processus d'abscission (Figure 2). D'un point de vue fonctionnel, quel bénéfice auraient les cellules filles à maintenir intact le pont intercellulaire tant que celui-ci est sous tension? Une première hypothèse serait d'aider, grâce au lien physique les reliant, la migration coordonnée des cellules filles, par exemple lors de la morphogenèse. Une seconde hypothèse serait de permettre aux cellules de rester mécaniquement connectées pendant leur réinsertion dans leur environnement après la division, assurant ainsi l'intégrité des tissus. $\diamond$ Mechanics and cell division: temporal control of abscission

\section{LIENS D'INTÉRÊT}

Les auteurs déclarent n'avoir aucun lien d'intérêt concernant les données publiées dans cet article.

\section{RÉFÉRENCES}

1. Hirano A, Kurimura T. Virally transformed cells and cytochalasin B. I. The effect of cytochalasin B on cytokinesis, karyokinesis and DNA synthesis in cells. Exp Cell Res 1974 ; 89 : 111-20.

2. Lacroix B, Maddox AS. Cytokinesis, ploidy and aneuploidy. J Pathol 2012 ; 226 : 338-51.

3. Yang D, Rismanchi N, Renvoisé B, et al. Structural basis for midbody targeting of spastin by the ESCRTIII protein CHMP1B. Nat Struct Mol Biol 2008 ; 15 : 1278-86.

4. Guizetti J, Schermelleh L, Mäntler J, et al. Cortical constriction during abscission involves helices of ESCT-III-dependent filaments. Science 2011 ; 331 : 1616-20.

5. Elia N, Sougrat R, Spurlin TA, et al. Dynamics of endosomal sorting complex required for transport (ESCRT) machinery during cytokinesis and its role in abscission. Proc Natl Acad Sci USA 2011 ; 108 : 484651.

6. Iwamori T, Iwamori N, Ma L, et al. TEXI4 interacts with CEP55 to block cell abscission. Mol Cell Biol $2010 ; 30$ : 2280-92.

7. Norden C, Mendoza M, Dobbelaere J, et al. The NoCut pathway links completion of cytokinesis to spindle midzone function to prevent chromosome breakage. Cell $2006 ; 125: 85-98$

8. Steigemann P, Wurzenberger C, Schmitz MHA, et al. Aurora B-mediated abscission checkpoint protects against tetraploidization. Cell 2009 ; $136:$ 473-84.

9. Carlton JG, Caballe A, Agromayor M, et al. ESCRT-III governs the Aurora B-mediated abscission checkpoint through CHMP4C. Science $2012 ; 336: 220-5$.

10. Lafaurie-Janvore J, Maiuri P, Wang I, et al. ESCRT-III Assembly and cytokinetic abscission are induced by tension release in the intercellular bridge. Science 2013 ; 339 : 1625-9.

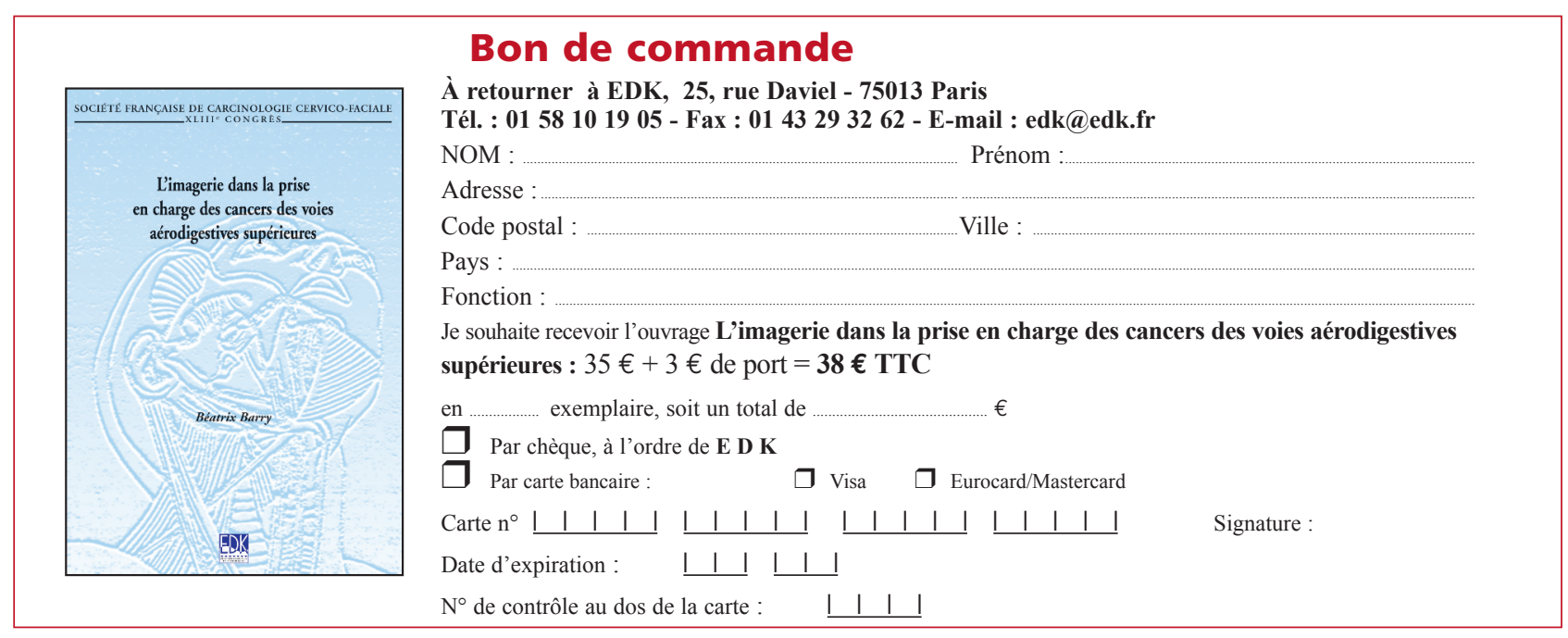

